\title{
Theoretical and experimental investigations on the nonlinear optical properties of gold(III) dithiolene complexes
}

\author{
I. Guezguez ${ }^{\text {a,b }}$, A. Karakas c , K. Iliopoulos ${ }^{\text {a,d,* }}$, B. Derkowska-Zielinska e , A. El-Ghayoury ${ }^{\text {a }}$, A. Ranganathan ${ }^{\text {a }}$, \\ P. Batail ${ }^{\text {a }}$, A. Migalska-Zalas ${ }^{\text {f }}$, B. Sahraoui ${ }^{\text {a,* }}$, M. Karakaya ${ }^{c}$ \\ a LUNAM Université, Université d'Angers, CNRS UMR 6200, Laboratoire MOLTECH-Anjou, 2 bd Lavoisier, 49045 Angers Cedex, France \\ ${ }^{\mathrm{b}}$ Laboratoire d'Electroniques et de Microélectroniques, Avenue de l'environnement 5019, Université de Monastir, Tunisie \\ ${ }^{\mathrm{c}}$ Selcuk University, Faculty of Sciences, Department of Physics Campus, Konya, Turkey \\ ${ }^{\mathrm{d}}$ Institute of Chemical Engineering Sciences, Foundation for Research and Technology Hellas (FORTH/ICE-HT), 26504 Patras, Greece \\ ${ }^{\mathrm{e}}$ Institute of Physics, Faculty of Physics, Astronomy and Informatics, Nicolaus Copernicus University, Grudziadzka 5, 87-100 Torun, Poland \\ f Instytut Fizyki, Katedra Fizyki Ciała Stałego Akademia im., Jana Długosza w Częstochowie, Poland
}

\section{A R T I C L E I N F O}

Article history:

Available online 6 June 2013

\section{Keywords:}

Nonlinear optical response

Dithiolene complexes

Degenerate four wave mixing

Time-dependent Hartree-Fock

\begin{abstract}
A B S T R A C T
Degenerate four-wave mixing (DFWM) experiments have been performed to determine the third-order nonlinear optical (NLO) susceptibilities $\left(\chi^{(3)}\right)$ of gold(III) maleimide dithiolate tetraphenylphosphonium, $\left(\mathrm{PPh}_{4}\right)\left[\mathrm{Au}(\mathrm{midt})_{2}\right],(\mathrm{Au}-\mathrm{P})$ and gold(III) maleimide dithiolate melamine melaminium hybrid solvate, $\left(\mathrm{C}_{3-}\right.$ $\left.\mathrm{N}_{6} \mathrm{H}_{6}\right)\left(\mathrm{C}_{3} \mathrm{~N}_{6} \mathrm{H}_{7}^{+}\right)\left[\mathrm{Au}(\text { midt })_{2}\right]^{-} \cdot 2 \mathrm{DMF} \cdot 2 \mathrm{H}_{2} \mathrm{O}$, (Au-Mel). Ab-initio quantum mechanical calculations (timedependent Hartree-Fock (TDHF) method) of Au-P and Au-Mel have been carried out to compute the electric dipole moment $(\mu)$, the dispersion-free and frequency-dependent dipole polarizability $(\alpha)$ and the second hyperpolarizability $(\gamma)$ values. These theoretical calculations are in good agreement with the experimentally obtained results by the DFWM technique. All the investigations show clearly the effect played by the counter ion on the resulting NLO properties of the two gold complexes.
\end{abstract}

(c) 2013 Elsevier B.V. All rights reserved.

\section{Introduction}

Nonlinear optical (NLO) materials play a major role as active components in a large range of applications such as optical communications, optical storage, optical computing, harmonic light generation, optical power limiting, optical rectifying devices, displays, printers, dynamic holography, frequency mixing and optical switching [1-6]. Bis-1,2-dithiolene complexes of transition metals have been widely investigated due to their combination of functional properties, specific geometries and intermolecular interactions. Due to their resulting interesting electronic properties, they have been used for the preparation of single component molecular metals $[7,8]$ and molecular conductors and superconductors $[9,10]$. These properties result from the extended delocalized $\pi$-system which makes them valuable candidates for photonic applications [11-18]. In addition, the resulting NLO properties can be modulated by changing the chemical structure of the dithiolate ligands, using different transition metals, modifying the oxidation state of the metal or even by changing the nature of the counter ion for the charged metal complexes. The incorporation of a heavy

* Corresponding authors at: LUNAM Université, Université d'Angers, CNRS UMR 6200, Laboratoire MOLTECH-Anjou, 2 bd Lavoisier, 49045 Angers Cedex, France. Tel.: +33 241735423 .

E-mail addresses: kostiliopoulos@gmail.com (K. Iliopoulos),bouchta.sahraoui@ univ-angers.fr (B. Sahraoui). atom introduces more sublevels into the energy hierarchy as compared to organic molecules with the same number of skeletal atoms, and this permits a greater number of allowed electronic transitions and hence enhances the NLO response. The central metal atom of a metal complex can readily be coordinated to conjugated ligands and undergo $\pi$-orbital overlap facilitating effective electronic communication. Since the potential of organic materials and metal complexes for NLO devices have been proven, the NLO properties of many of these compounds have been investigated by both experimental and theoretical methods $[19,20]$. We have recently reported the preparation of two gold dithiolene metal complexes which differ by the nature of the counter cations be they a phosphonium (Au-P) or melaminium (Au-Mel) [21]. In these complexes, we have structurally shown in the case of the melaminium (Au-Mel) the presence of charge-assisted hydrogenbonded cation-anion interaction in the solid state. The investigation of the NLO properties of the two gold dithiolene metal complexes by means of $Z$-scan showed a significant modification of the NLO response suggesting a clear effect of the counter ion on the NLO properties. More specifically, we have demonstrated an enhancement of the second order hyperpolarizability (by about a factor of 2.5) in the case of the hydrogen bonded melaminium counter cation [22].

In this work, we report the experimental and theoretical investigation of the third-order NLO properties for the two gold(III) 
dithiolene complexes Au-P and Au-Mel (Fig. 1). The third-order NLO susceptibilities of these materials in tetrahydrofuran (THF) have been determined experimentally by the DFWM technique and the measured nonlinear susceptibilities and the electronic absorption behavior are compared by quantum mechanical calculations. Moreover our findings are compared with our previous results [22] as well as other previously published reports.

\section{Experimental}

The Au-P and Au-Mel metal complexes have been prepared as previously reported [21]. The transmission spectra were measured using a Perkin-Elmer UV/Vis Lambda 2 spectrometer in the 300 $1000 \mathrm{~nm}$ spectral region at room temperature.

The $\chi^{(3)}$ was measured by means of the DFWM method using a Nd:YAG laser (Quantel Model YG472) working at $532 \mathrm{~nm}$ with 30 ps pulses duration and $1 \mathrm{~Hz}$ repetition rate. We used the standard backward DFWM geometry described elsewhere $[23,24]$.

The DFWM method can provide the absolute value of the third order nonlinear susceptibility, which in general consists of a real and imaginary part, which are related to the nonlinear refraction and absorption respectively.

The DFWM reflectivity $(R)$ was calculated from the propagation equations of the interacted beams, which were deduced from Maxwell's equations using the slowly varying amplitude approximation, taking into account only the linear absorption coefficient. The DFWM reflectivity $(R)$, defined as the total DFWM signal intensity divided by the incident probe-pulse intensity, is found as a function of the pump-pulse intensity. The probe intensity $I_{3}$ was adjusted to be approximately $6 \times 10^{-2} I_{1}$. The DFWM reflectivity $(R)$ can be expressed as follows [23,24]:

$R=\frac{I_{4}(0)}{I_{3}(0)}=\left(\frac{48 \pi^{3}}{n^{2} c \lambda} \chi^{(3)}\right)^{2} \frac{I_{1}(0) I_{2}(0) \exp (-\Gamma L)}{\left[p \operatorname{coth}(p L)+\frac{\Gamma}{2}\right]^{2}}$

where

$p^{2}=\frac{\Gamma^{2}}{4}-\left(\frac{48 \pi^{3}}{n^{2} c \lambda} \chi^{(3)}\right)^{2} I_{1}(0) I_{2}(0)$

The parameters $\Gamma, L, \lambda, \mathrm{n}$ and $\mathrm{c}$ are the linear absorption coefficient, the thickness of the sample, the laser wavelength used, the linear refractive index of the considered material and the velocity of light in vacuum, respectively. $I_{1}(0)$ and $I_{2}(0)$ are the intensity of the pumping beams, $I_{3}(0)$ is the probe beam, and $I_{4}(0)$ is the phase-conjugated beam. Carbon disulfide $\left(\mathrm{CS}_{2}\right)$ was used as a reference material during the measurements.

\section{Theoretical calculations}

The theoretical computations involve the determination of electric dipole moments $(\mu)$, dispersion-free and frequency-dependent dipole polarizability $(\alpha)$ and second hyperpolarizability $(\gamma)$ tensor components. The molecular geometries of Au-P and Au-Mel have been firstly optimized in the restricted close-shell Hartree-Fock (RHF) level. Actually, the optimized geometries through ab initio calculations closely match the geometries obtained from the $\mathrm{X}$-ray analysis. The geometry optimization has been followed by the calculations of electric dipole moments and (hyper)polarizabilities with SBKJC (Stevens/Basch/Krauss/Jasien/Cundari) valence basis set. In transition metals, the ab initio effective core potentials (ECPs) usually include the semicore $n s$ and $n p$ electrons in the valence set, necessitating a correspondingly larger basis set. The SBKJC basis set is appropriate for the calculations of (hyper)polarizabilities of the examined complexes with rather heavy gold(III) ions. $\mu$, static and dynamic $\alpha$ and $\gamma$ computations have been performed using the SBKJC valence basis set with corresponding ECPs. The theoretical understanding of the NLO properties is growing during the last years and several tested computational codes are important as valuable theoretical tools used for the computation of the (hyper)polarizabilities. Frequency-dependent (hyper)polarizabilities have been frequently implemented at the self-consistent field level of theory (known as TDHF) [25]. $\alpha(0 ; 0)$, $\gamma(0 ; 0,0,0)$ at $\omega=0$ and $\alpha(-\omega ; \omega), \gamma(-3 \omega ; \omega, \omega, \omega)$ calculations at $\omega=0.0856252$ atomic units (a.u.) (i.e. at $\lambda=532 \mathrm{~nm}$ wavelength) according to the laser frequency used in the DFWM measurements have been carried out using the TDHF method with the SBKJC basis set implemented in the GAMESS [26] program. The $\alpha(0 ; 0)$ and $\gamma(0 ; 0,0,0)$ definitions mentioned above describe the static dipole polarizability and third-order hyperpolarizability, respectively. The calculations of $\gamma(-3 \omega ; \omega, \omega, \omega)$ were carried out by the third-harmonic generation (THG) group of the TDHF procedure. The TDHF procedure gives useful predictive values and can be of direct interest for understanding both static and dynamic (hyper)polarizabilities of organic molecules and transition metal complexes.

The orientationally averaged (isotropic) dipole polarizability $\langle\alpha\rangle$ and third-order hyperpolarizability $\langle\gamma\rangle$ values have been calculated using the following expressions, respectively [27]:

$$
\langle\alpha\rangle=\left(\alpha_{x x}+\alpha_{y y}+\alpha_{z z}\right) / 3
$$

$\langle\gamma\rangle=(1 / 5)\left[\gamma_{x x x x}+\gamma_{y y y y}+\gamma_{z z z z}+2\left(\gamma_{x x y y}+\gamma_{x x z z}+\gamma_{y y z z}\right)\right]$

We have also computed the $\chi^{(3)}(-\omega ; \omega,-\omega, \omega)$ susceptibility tensors which represent the nonlinear interaction of third-order. The frequency-dependent orientationally averaged (isotropic) $\left\langle\chi^{(3)}(-\omega ; \omega,-\omega, \omega)\right\rangle$ calculations at $\omega=0.0856252$ a.u. were carried out by the Optical Kerr Effect (OKE) group of the TDHF method with the SBKJC basis set implemented in the GAMESS [26] program.

To calculate all electric dipole moments, (hyper)polarizabilities and susceptibilities, the origin of cartesian coordinate system $(x, y, z)=(0,0,0)$ has been chosen at the centers of mass of Au-P and Au-Mel. All electric dipole moment, (hyper)polarizability and susceptibility calculations have been performed on a PC with an Intel (R) core (TM) I7-2630QM operator, 5.8 GB RAM memory and $2 \mathrm{GHz}$ frequency using Linux PC GAMESS version running under Linux Fedora release 11 (Leonidas) environment.

Besides, the transition wavelengths $(\lambda)$ of the lowest lying electronic transitions together with the corresponding excitation

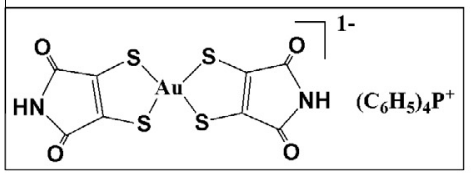

$\mathrm{Au}-\mathrm{P}$

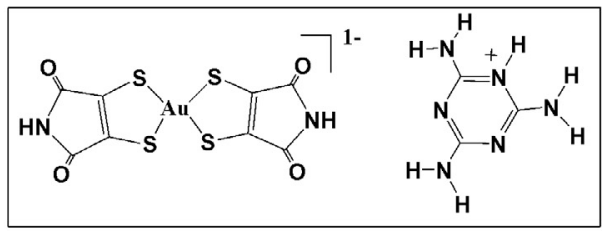

$\mathrm{Au}-\mathrm{Mel}$

Fig. 1. Chemical structures of the gold(III) dithiolene metal complexes. 
energies and oscillator strengths for the Au-P and Au-Mel dithiolene complexes have been determined theoretically by the TDHF method with the CEP-4G basis set implemented in the GAUSSIAN03W [28] program. To understand the relationship of the NLO properties with the molecular structure HOMOs, LUMOs and HOMO-LUMO gaps have been also generated in the same theoretical framework as the electronic transition wavelength calculations using GAUSSIANO3W [28] program.

\section{Results and discussion}

\subsection{UV-Vis spectroscopy and DFWM measurements}

The electronic absorption spectra of Au-P and Au-Mel were recorded in THF solution (Fig. 2). These complexes exhibit a strong electronic absorption band at $\lambda=288 \mathrm{~nm}$ which is assigned to the ligand centered $n \rightarrow \pi$ transition. The broad band observed in the visible region around $\lambda_{\max }=447 \mathrm{~nm}$ is characteristic of $d-p$. The measured linear absorption coefficients are listed in Table 1.

The NLO properties of Au-Mel and Au-P dissolved in THF were investigated using the DFWM method at $532 \mathrm{~nm}$. Typical plots of the DFWM reflectivity $(R)$ versus the input intensity $\left(I_{1}\right)$ of the Au-Mel and Au-P are presented in Fig. 3. During the DFWM experiments, the quadratic dependence of DFWM reflectivity on the input intensity was verified for all samples. Fig. 3 also presents the theoretical fitting results of the DFWM reflectivity $(R)$ data recorded for the studied samples at $532 \mathrm{~nm}$. From the dependencies of the experimental data and Eq. (1), we derived the absolute values of $\chi^{(3)}$, which are presented in Table 2.

The $\chi^{(3)}$ of Au-Mel was found to be larger than that of Au-P by a factor of 1.7 (Table 2) and is consistent with the charge-assisted hydrogen bonding interaction identified in the case of Au-Mel. This result is in perfect agreement with the theoretical calculations presented in this work (see also Table 2 ) and in very good agreement with our recent $Z$-scan findings [22], as the corresponding ratio of the second order hyperpolarizabilities determined in Ref. [22] has been found to be 2.5 . The differences between the $Z$-scan and the DFWM measurements can be attributed to the different solvent utilized. Moreover with the $Z$-scan technique the real and imaginary part of the nonlinearity can be separately measured, while in the DFWM measurements the nonlinear absorption cannot be determined. This can have a large impact in the different values found in the two investigations as the nonlinear absorption has been found to be as significant as the nonlinear refraction [22]

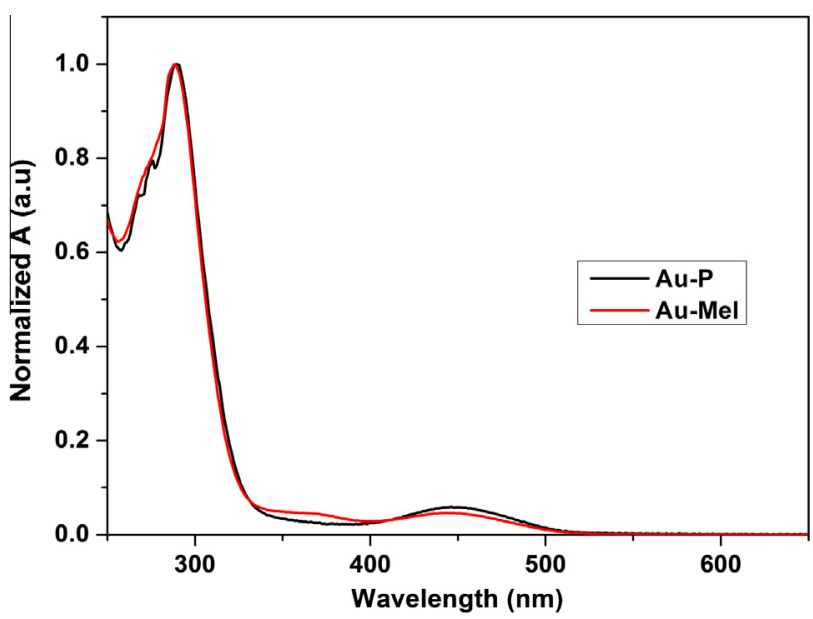

Fig. 2. UV-Vis absorption spectra of Au-Mel and Au-P in THF $\left(C=10^{-5} \mathrm{~mol} / \mathrm{L}\right)$.
Table 1

Measured linear absorption coefficient $\Gamma(\mathrm{cm})^{-1}$ values of Au-Mel and Au-P at $532 \mathrm{~nm}$, corresponding to a concentration of $1.4 \mathrm{~g} / \mathrm{L}$.

\begin{tabular}{ll}
\hline Complex & $\Gamma$ \\
\hline Au-Mel & 2.4 \\
Au-P & 1.8 \\
\hline
\end{tabular}

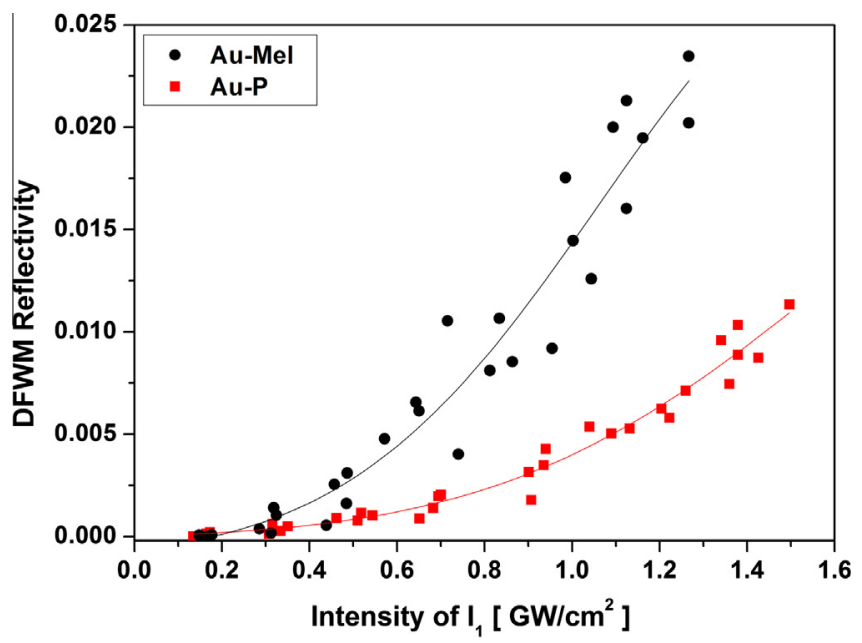

Fig. 3. DFWM reflectivity of Au-Mel and Au-P dissolved in THF ( $C=1.4 \mathrm{~g} / \mathrm{L})$ as a function of the intensity of the input pump beam $I_{1}$ at $532 \mathrm{~nm}$. Solid lines show the best fit of Eq. (2) to the experimental data.

Table 2

Measured $\chi^{(3)}$ and computed $\left\langle\chi^{(3)}(-\omega ; \omega,-\omega, \omega)\right\rangle$ values at $\omega=0.0856252$ a.u. for the Au-P and Au-Mel dithiolene complexes, corresponding to a concentration of $1.4 \mathrm{~g} / \mathrm{L}$.

\begin{tabular}{lll}
\hline Complex & Au-P & Au-Mel \\
\hline$\chi^{(3)}\left(\times 10^{-13}\right.$ esu $)$ (measured) & 3.864 & 6.727 \\
$\left\langle\chi^{(3)}\right\rangle\left(\times 10^{-13}\right.$ esu) (calculated) & 4.603 & 8.005 \\
\hline
\end{tabular}

Table 3

The ab initio calculated electric dipole moments $\mu$ (Debye) and dipole moment components for Au-P and Au-Mel.

\begin{tabular}{lrlrr}
\hline Complex & \multicolumn{1}{l}{$\mu_{x}$} & $\mu_{y}$ & \multicolumn{1}{c}{$\mu_{z}$} & \multicolumn{1}{c}{$\mu$} \\
\hline Au-P & 2.128 & 0.026 & 2.889 & 3.588 \\
Au-Mel & -30.034 & 0.010 & -0.707 & 30.043 \\
\hline
\end{tabular}

Table 4

Some selected components of the static $\alpha(0 ; 0)$ and $\langle\alpha\rangle(0 ;)\left(\times 10^{-24}\right.$ esu $)$ values for Au$\mathbf{P}$ and Au-Mel.

\begin{tabular}{llllr}
\hline Complex & $\alpha_{x x}$ & $\alpha_{y y}$ & $\alpha_{z z}$ & \multicolumn{1}{c}{$\langle\alpha\rangle$} \\
\hline Au-P & 125.664 & 46.524 & 47.641 & 73.276 \\
Au-Mel & 276.847 & 30.658 & 25.976 & 111.160 \\
\hline
\end{tabular}

Table 5

Some selected components of the frequency-dependent $\alpha(-\omega ; \omega)$ and $\langle\alpha\rangle(-\omega ; \omega)$ $\left(\times 10^{-24} \mathrm{esu}\right)$ values at $\omega=0.0856252$ a.u. for Au-P and Au-Mel.

\begin{tabular}{lllll}
\hline Complex & $\alpha_{x x}$ & $\alpha_{y y}$ & $\alpha_{z z}$ & $\langle\alpha\rangle$ \\
\hline Au-P & 120.377 & 67.513 & 52.711 & 80.200 \\
Au-Mel & 68.269 & 33.539 & 40.896 & 47.568 \\
\hline
\end{tabular}


Table 6

Some selected components of the static $\gamma(0 ; 0,0,0)$ and $\langle\gamma\rangle(0 ; 0,0,0)\left(\times 10^{-37}\right.$ esu $)$ values for Au-P and Au-Mel.

\begin{tabular}{llrrrr}
\hline & $\gamma_{x x x x}$ & \multicolumn{1}{c}{$\gamma_{y y y y}$} & \multicolumn{1}{c}{$\gamma_{z z z z}$} & \multicolumn{1}{c}{$\gamma_{x x y y}$} & \multicolumn{1}{c}{\langle} \\
\hline Au-P & 438325.653 & 1208.944 & 2350.623 & 17928.523 & 405.039 \\
Au-Mel & 126160.445 & 568.202 & 112.984 & 1371.143 & 98008.398 \\
\hline
\end{tabular}

\subsection{Computational results and discussion}

Quantum chemical calculations have been shown to be useful in the description of the relationship between the electronic structure of the systems and its NLO response [29]. The computational approach allows the determination of molecular NLO properties as an inexpensive way to design molecules by analyzing their potential before synthesis and to determine high-order hyperpolarizability tensors of molecules. The hyperpolarizability tensors of the molecules could be determined using a suitable computational approach. These tensors describe the response of molecules to an external electric field. At the molecular level, the NLO properties are determined by their dynamic hyperpolarizabilities. TDHF is a procedure generally used to find out approximate values, and can be a mean of understanding both the static and dynamic hyperpolarizabilities of organic molecules and transition metal complexes. In this work, the orientationally averaged (isotropic) values of third-order susceptibilities for Au-P and Au-Mel have been computed. Both the measured $\chi^{(3)}$ and calculated $\left\langle\chi^{(3)}\right\rangle$ values show that the third order nonlinear susceptibility is higher in the case of the Au-Mel molecular system. In this sense it is obvious that changing the cation from tetraphenylphosphonium to melamine melaminium hybrid has generated almost a $100 \%$ increase on the measured and calculated third-order susceptibilities (Table 2). In this study, in addition to the static dipole polarizabilities $\alpha(0 ; 0)$
Table 9

Calculated HOMO-LUMO energy (a.u.) and HOMO-LUMO band gap $\left(E_{g}\right)$ values for Au-P and Au-Mel

\begin{tabular}{lrr}
\hline & \multicolumn{1}{c}{ Au-P } & \multicolumn{1}{c}{ Au-Mel } \\
\hline HOMO & -0.30194 & -0.30935 \\
LUMO & -0.00442 & -0.01986 \\
$E_{g}[$ HOMO-LUMO] & 0.29752 & 0.28949 \\
HOMO-1 & -0.33207 & -0.34460 \\
LUMO + 1 & 0.01562 & -0.01778 \\
$E_{g}[($ HOMO-1)-(LUMO + 1)] & 0.34769 & 0.32682 \\
\hline
\end{tabular}

and second hyperpolarizabilities $\gamma(0 ; 0,0,0)$, the following processes for dynamic (hyper)polarizabilities have been considered: frequency-dependent dipole polarizabilities $\alpha(-\omega ; \omega)$ and second hyperpolarizabilities $\gamma(-3 \omega ; \omega, \omega, \omega)$. The electric dipole moments, some significant calculated magnitudes of the static and dynamic dipole polarizabilities and second hyperpolarizabilities are shown in Tables 3-7. The $\gamma$ values depend on a number of factors, which include the extent of $\pi$-electron conjugation, the dimensionality of the molecules and the nature of substituents. The introduction of transition metals with partially filled $d$-shells is known to affect a number of charge transfer (CT) mechanisms such as metal-ligand charge transfer (MLCT), ligand-metal charge transfer (LMCT) and $d-d$ charge transfer [30]. The metal ions of gold complexes are best described as $d^{8}$, as expected for a square planar

Table 7

Some selected components of the frequency-dependent $\gamma(-3 \omega ; \omega, \omega, \omega)$ and $\langle\gamma\rangle(-3 \omega ; \omega, \omega, \omega)\left(\times 10^{-37}\right.$ esu) values at $\omega=0.0856252$ a.u. for Au-P and Au-Mel

\begin{tabular}{|c|c|c|c|c|c|c|}
\hline & $\gamma_{x x x x}$ & $\gamma_{y y y y}$ & $\gamma_{z z z z}$ & $\gamma_{x x z z}$ & $\gamma_{y y z z}$ & $\langle\gamma\rangle$ \\
\hline Au-P & -5144.818 & 7657.435 & -6203.664 & 35960.561 & -4706.753 & 8784.124 \\
\hline Au-Mel & 1065030.436 & 3371.988 & -81041.707 & 375413.540 & 4206.268 & 519899.692 \\
\hline
\end{tabular}

Table 8

Calculated maximum OPA wavelengths $\lambda(\mathrm{nm})$, excitation energies $(\mathrm{eV})$ and oscillator strengths $(f)$ for $\mathbf{A u}-\mathbf{P}$ and $\mathbf{A u}-\mathbf{M e l}$.

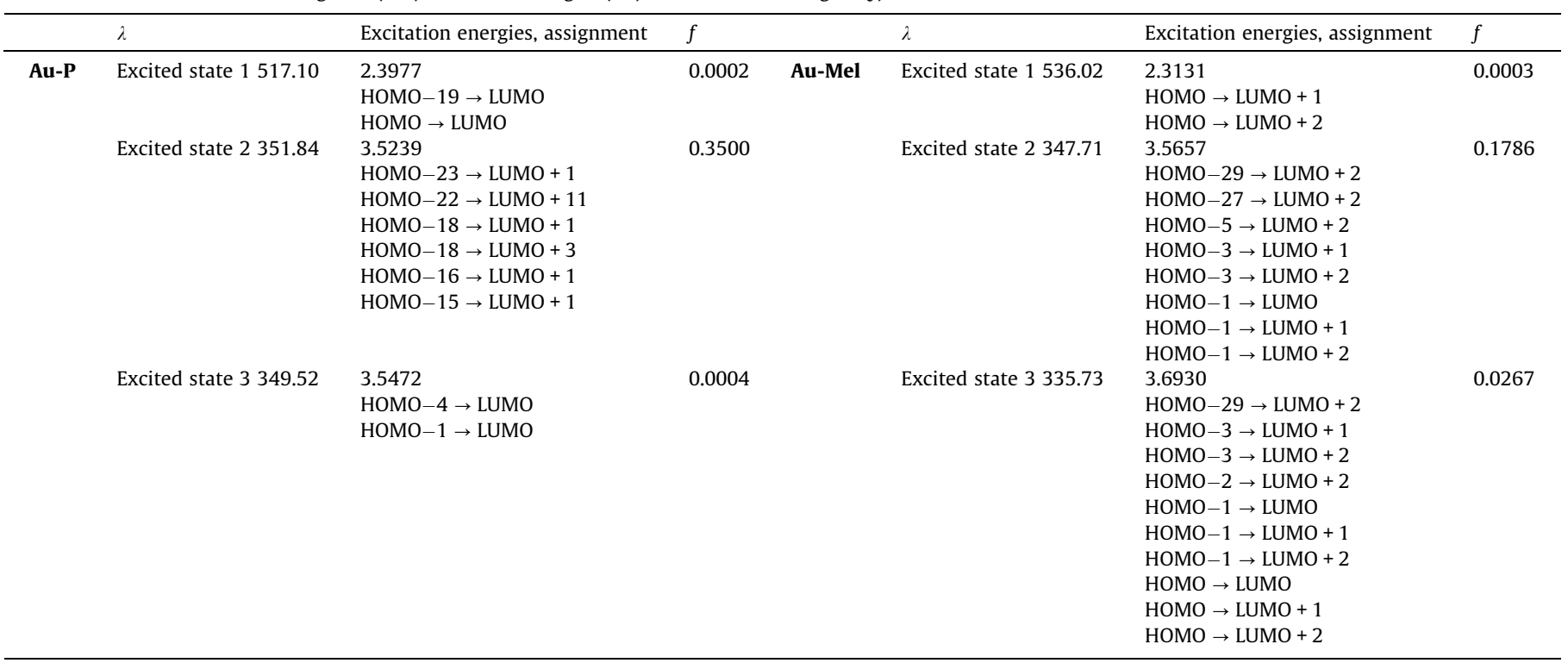


gold(III) compound, and therefore the unpaired electron in the molecule can reside largely on the ligands. The square planar gold(III) dithiolene complexes Au-P and Au-Mel have partially filled $d$-shells, which are also in favor of higher values of nonlinear optical response. In addition, the involvements of $\mathrm{Au}(\mathrm{III})$ ions with $d^{8}$ electron structures for square planar $\mathrm{Au}(\mathrm{III})$ complexes here result in obvious enhancement of all the molecular (hyper)polarizabilities. The $\mu$ and dynamic $\langle\gamma(-3 \omega ; \omega, \omega, \omega)\rangle$ values have the same enhancement in the order Au-P $\left\langle\right.$ Au-Mel as the $\chi^{(3)}$ and $\left\langle\chi^{(3)}\right\rangle$ values (see Tables 2, 3, and 7).

The calculated wavelengths $(\lambda)$ and oscillator strengths $(f)$ for the maximum electronic absorption of Au-P and Au-Mel are shown in Table 8. As can be seen, the optical spectra of Au-P and Au-Mel, respectively, exhibit three weak bands centered between
349 and $517 \mathrm{~nm}$ for Au-P and between 335 and $536 \mathrm{~nm}$ for AuMel. The transitions centered at $351.84 \mathrm{~nm}$ for Au-P and $347.71 \mathrm{~nm}$ for Au-Mel of absorption maxima are relatively stronger with 0.3500 and 0.1786 oscillator strengths, respectively, than the others with almost zero values of oscillator strengths. Both these intense bands theoretically obtained at $351.84 \mathrm{~nm}$ and $347.71 \mathrm{~nm}$ for Au-P and Au-Mel, respectively, while experimentally observing at $288 \mathrm{~nm}$ in the UV region, are located in the UV region assigning to $n \rightarrow \pi$ transitions. Theoretically above $517 \mathrm{~nm}$ and $536 \mathrm{~nm}$ for Au-P and Au-Mel, respectively, and also experimentally above $447 \mathrm{~nm}$ no additional absorption band is observed in the visible region.

To understand the phenomenon in the context of the molecular orbital picture, on the basis of optimized geometries, we examined

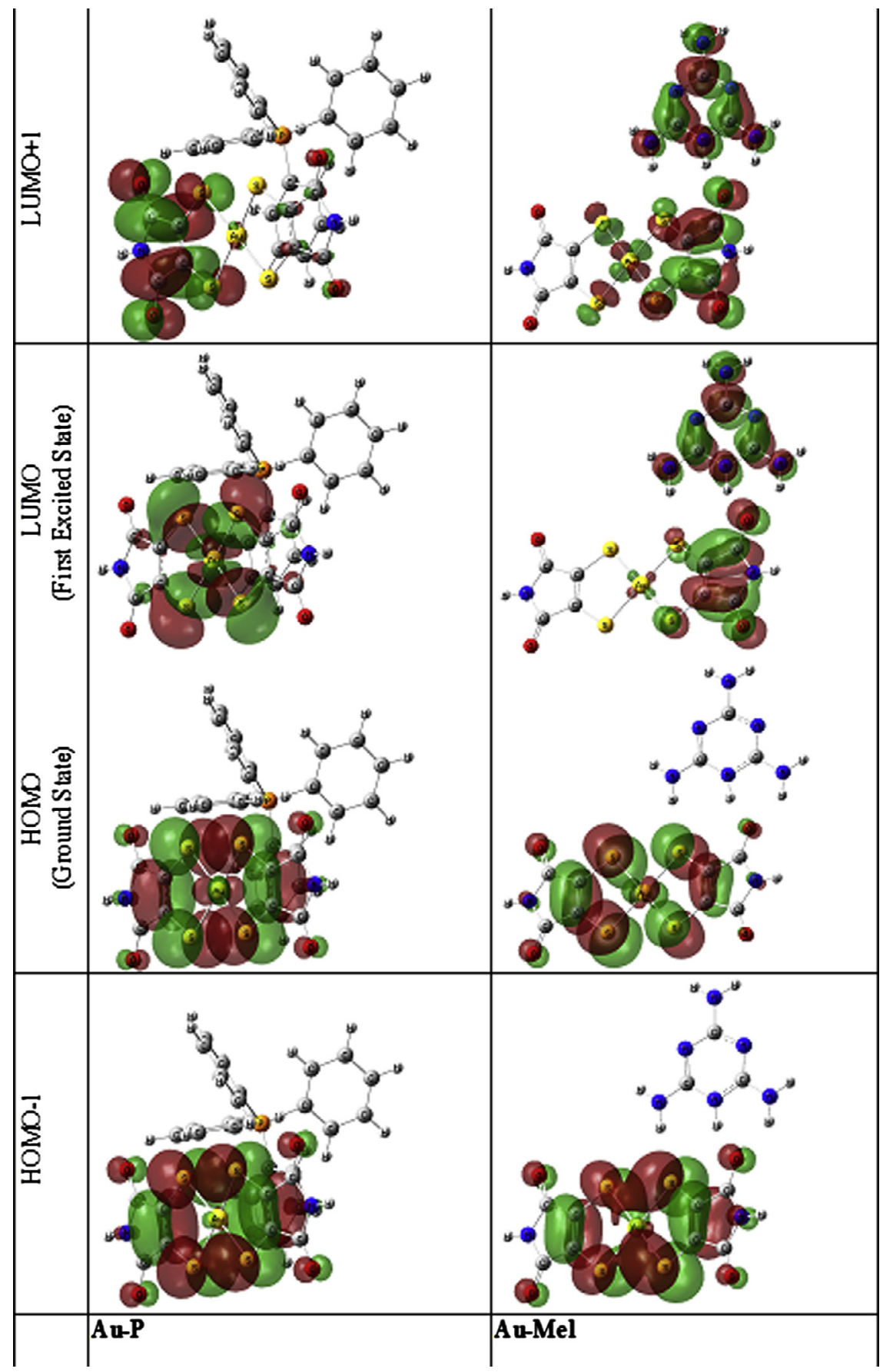

Fig. 4. The frontier and second frontier molecular orbitals of Au-P and Au-Mel. 
the molecular HOMO and LUMO energies of the electronic structures. The calculated HOMO and LUMO energies and HOMO-LUMO energy gaps of Au-P and Au-Mel are listed in Table 9. The frontier molecular orbitals associated with the optical transitions are shown in Fig. 4. The second and third-order NLO responses can be dictated by CT excitations involving the HOMO and LUMO orbitals in such a way that the larger values of first and second hyperpolarizabilities should correspond to lower HOMO-LUMO gaps. The hyperpolarizabilities are therefore directly related to the HOMO-LUMO energy gap. It is evident that there should be an inverse relationship between the HOMO-LUMO gap and hyperpolarizability values [31]. The best values of hyperpolarizabilities can be achieved with lower HOMO-LUMO gaps. Therefore, it could be expected that Au-Mel with a bit lower HOMO-LUMO gap than that of Au-P might give much higher value for dynamic $\langle\gamma(-3 \omega ; \omega, \omega, \omega)\rangle$ (see Tables 7 and 9). For Au-P, the electron density is located mainly on the anion part in the HOMO and nearby occupied molecular orbitals and also in the LUMO and nearby unoccupied molecular orbitals. For Au-Mel, the electron density is located on the anion part in the HOMO, whereas the electron density on the LUMO and nearby unoccupied molecular orbitals is mainly localized on the cation part (Fig. 4). These results are in good agreement with the electronic delocalization occurring through the presence of the charge-assisted hydrogen bonding interaction in Au-Mel, while such interaction is absent in the Au-P complex. The HOMO-LUMO gap may be decreased by the introduction of hydrogen-bonded interaction in the molecular structures as has been reported by Liao [32] where he showed that the presence of hydrogen bonding is controlled predominately by the HOMOLUMO gap. Our theoretical results on hydrogen bonding effect and HOMO-LUMO gap (Table 9, Fig. 4) follow the same tendency as observed by Liao. Au-Mel with charge-assisted hydrogen bonding interaction has, thus, a slightly higher HOMO energy and a slightly lower HOMO-LUMO band gap than that of Au-P.

\section{Conclusions}

The third-order optical nonlinearities of Au-P and Au-Mel have been measured by the DFWM technique. The OPA characterizations of Au-P and Au-Mel have been obtained both experimentally (UV-Vis spectroscopy) and theoretically (TDHF method). A comparison between theory and experiment for linear optical spectra is straightforward as the regions of absorption and transparency predicted by theory match with the experimental values well. This confirms that, especially for the transition metal complexes, TDHF is one of the best methods for estimating the electron absorption spectra. To better understand the NLO enhancements in gold(III) complexes here, we examined the frontier orbitals. Au-P and AuMel show small HOMO-LUMO gaps, and thus produce the microscopic second hyperpolarizabilities with non-zero values. The third-order NLO properties have been also studied theoretically. Theoretical results for the (hyper)polarizabilities and susceptibilities derived from ab initio quantum mechanical calculations explain the experimental findings. The computed data in this work have been compared with previous published results in the literature.

\section{Acknowledgements}

K.I acknowledges support from the European Commission and General Secretariat for Research and Technology (Greece) for a National Strategic Reference Framework (NSRF) Project (PE3-(1612)).

\section{References}

[1] F. Kajzar, J.D. Swalan (Eds.), Organic Thin Films for Waveguiding Nonlinear Optics, Gordon and Breach, Publishers, 1996.

[2] M.G. Kuzyk, C.W. Dirk (Eds.), Characterization Techniques and Tabulations for Organic Nonlinear Optical Materials, Marcel Dekker Inc., 1998.

[3] J.V. Moloney, Nonlinear Optical Materials, Springer, New York, 1998

[4] B. Sahraoui, G. Rivoire, J. Zaremba, N. Terkia-Derdra, M. Sallé, J. Opt. Soc. Am. B 15 (1998) 923.

[5] O. Krupka, A. El-Ghayoury, I. Rau, B. Sahraoui, J.G. Grote, F. Kajzar, Thin Solid Films 516 (2008) 8932.

[6] I. Fuks-Janczarek, J. Luc, B. Sahraoui, F. Dumur, P. Hudhomme, J. Berdowski, I.V. Kityk, J. Phys. Chem. B 109 (2005) 10179.

[7] H. Tanaka, Y. Okano, H. Kobayashi, W. Suzuki, A. Kobayashi, Science 291 (2001) 285.

[8] A. Kobayashi, E. Fujiwara, H. Kobayashi, Chem. Rev. 104 (2004) 5243.

[9] A.E. Underhill, J. Mater. Chem. 2 (1992) 1.

[10] R.P. Shibaeva, E.B. Yagubskii, Chem. Rev. 104 (2004) 5347

[11] C.S. Winter, C.A.S. Hill, A.E. Underhill, Appl. Phys. Lett. 58 (1991) 107.

[12] Z. Dai, X. Yue, B. Peng, O. Yang, X. Liu, P. Ye, Chem. Phys. Lett. 317 (2000) 9.

[13] G. Chatzikyriakos, I. Papagiannouli, S. Couris, G.C. Anyfantis, G.C. Papavassiliou, Chem. Phys. Lett. 513 (2011) 229.

[14] L. Hu, J. Qin, N. Zhou, Y.-F. Meng, Y. Xu, J.-L. Zuo, X.-Z. You, Dyes Pigments 92 (2012) 1223.

[15] P. Aloukos, G. Chatzikyriakos, I. Papagiannouli, N. Liaros, S. Couris, Chem. Phys. Lett. 495 (2010) 245.

[16] G. Soras, N. Psaroudakis, G.A. Mousdis, M.J. Manos, A.J. Tasiopoulos, P. Aloukos, S. Couris, P. Labéguerie, J. Lipinski, A. Avramopoulos, M.G. Papadopoulos, Chem. Phys. 372 (2010) 33.

[17] G.C. Anyfantis, G.C. Papavassiliou, P. Aloukos, S. Couris, Y.F. Weng, H. Yoshimo, K. Murata, Z. Naturforsch 62 (2007) 200.

[18] P. Aloukos, S. Couris, J.B. Koutselas, G.C. Anyfantis, G.C. Papavassiliou, Chem. Phys. Lett. 428 (2006) 109.

[19] H.S. Nalwa, M. Hanack, G. Pawlowski, M.K. Engel, Chem. Phys. 245 (1999) 17.

[20] K. Iliopoulos, R. Czaplicki, H. El Ouazzani, J.Y. Balandier, M. Chas, S. Goeb, M. Sallé, D. Gindre, B. Sahraoui, Appl. Phys. Lett. 97 (2010) 101104.

[21] A. Rangananthan, A. El-Ghayoury, L. Zorina, P. Batail, Cryst. Eng. Commun. 12 (2010) 4268.

[22] K. Iliopoulos, A. El-Ghayoury, B. Derkowska, A. Ranganathan, P. Batail, D. Gindre, B. Sahraoui, Appl. Phys. Lett. 101 (2012) 261105.

[23] B. Derkowska, M. Wojdyła, W. Bała, K. Jaworowicz, M. Karpierz, J.G. Grote, O. Krupka, F. Kajzar, B. Sahraoui, J. Appl. Phys. 101 (2007) 083112.

[24] B. Derkowska, in: James L. Davies, Daniel A. Hall (Eds.), Nonlinear Optics Research Progress, Nova Science Publishers Inc., 2008, pp. 189-222.

[25] D.P. Shelton, J.E. Rice, Chem. Rev. 94 (1994) 3.

[26] Intel $\times 86$ (win32, Linux, OS/2, DOS) version. PC GAMESS version 6.2, build number 2068. This version of GAMESS is described in: M.W. Schmidt et al., J. Comput. Chem. 14 (1993) 1347.

[27] M.P. Bogaard, B.J. Orr, A.D. Buckingham (Eds.), MTP International Review of Science, Butterworths, London, 1975. vol. 2, p. 149.

[28] M.J. Frisch et al. Gaussian 03, Revision E.01, Gaussian, Inc., Wallingford CT, 2004.

[29] D.M. Burland, R.D. Miller, C.A. Walsh, Chem. Rev. 94 (1994) 31.

[30] K.P. Unnikrishnan, J. Thomas, V.P.N. Nampoori, C.P.G. Vallabhan, Synth. Met. 139 (2003) 371.

[31] P.S. Liyanage, R.M. de Silva, K.M. Nalin de Silva, J. Mol. Struct. (Theochem) 639 (2003) 195.

[32] H.-Yi. Liao, J. China Chem. Soc. 56 (3) (2009) 532. 